\title{
Morphometric analysis of the relationships between the maxillary first molar and maxillary sinus floor
}

\author{
Andreea Didilescu ${ }^{1,2^{*}}$, Mugurel Rusu $^{3}$, Mihai Săndulescu ${ }^{4}$, Carmen Georgescu $^{5}$, Radu Ciuluvică ${ }^{3}$ \\ ${ }^{1}$ Department of Anatomy, Faculty of Medicine and Pharmacy, Dunarea de Jos University, Galati, Romania \\ ${ }^{2}$ Department of Embryology, Faculty of Dental Medicine, Carol Davila University of Medicine and Pharmacy, Bucharest, Romania \\ ${ }^{3}$ Department of Anatomy, Faculty of Dental Medicine, Carol Davila University of Medicine and Pharmacy, Bucharest, Romania \\ ${ }^{4}$ Department of Oral Implantology, Faculty of Dental Medicine, Carol Davila University of Medicine and Pharmacy, Bucharest, Ro- \\ mania \\ ${ }^{5}$ Department of Prosthodontics Technology and Dental Materials, Faculty of Dental Medicine, Carol Davila University of Medicine \\ and Pharmacy, Bucharest, Romania \\ Email: ${ }^{*}$ andreea.didilescu@gmail.com
}

Received 16 October 2012; revised 20 November 2012; accepted 28 November 2012

\begin{abstract}
Objectives: To assess the relationships between the maxillary first molar and the maxillary sinus floor in a group of patients referred to a dental clinic. Methods: Ninety-seven patients were recruited for this study. The distances between the examined roots (mesio-buccal, disto-buccal and palatal) as well as furcations, and the sinus floor, were evaluated using cone beam computed tomography, and grouped as follows: class 0: distance $=0 \mathrm{~mm}$; class $1: 0 \mathrm{~mm}<$ distance $<2 \mathrm{~mm}$; class 2: $2 \mathrm{~mm} \leq$ distance $<4 \mathrm{~mm}$; class 3: $4 \mathrm{~mm} \leq$ distance $<6 \mathrm{~mm}$; class $4: 6 \mathrm{~mm} \leq$ distance. The Spearman's Rank Correlation coefficient was used to test the univariate associations between furcation-sinus floor distance and each root class. Results: The prevalence of class 0 was the highest for the palatal root $(44.33 \%)$, followed in descending order by mesio-buccal $(40.21 \%)$, and disto-buccal $(38.14 \%)$ roots. The highest correlation coefficient was recorded when assessing the relationship between furcation-sinus floor distance and palatal root classes $(r h o=0.66, p<0.001, n=97)$. Conclusions: Altogether, the results suggest that the palatal root of the maxillary first molar not only had the closest relationship with the sinus floor, but also proved to be the best predictor for the furcation-sinus floor distance. The clinician should be aware of the anatomical and morphological details of this root, especially when taking surgical decisions.
\end{abstract}

Keywords: Cone-Beam Computed Tomography; Maxillary Sinus; Endodontics; Oral Surgical Procedures

\footnotetext{
"Corresponding author.
}

\section{INTRODUCTION}

The development of the maxillary sinus begins during fetal period and continues after birth. With the completion of the adult dentition eruption sequence, the maxillary sinus reaches its full development. The maxillary sinus is a pyramid-shaped osseous cavity, the base being represented by the nasal antral wall and the tip lying in the zygomatic bone. Its volume is estimated to be about an average of $15 \mathrm{cc}$. [1]. The inside lining, a ciliary epithelium, transports bacteria and other possible foreign material toward the opening to the nasal cavity (maxillary ostium) on a thin mucous layer. A healthy maxillary sinus is aseptic in $80 \%$ to $100 \%$ of the population, containing neither bacteria nor any other foreign material $[2,3]$. The inferior wall of the maxillary sinus, which is curved rather than flat, is formed by the lower third of the medial wall and the buccoalveolar wall. The topography of the inferior wall with the maxillary root apices varies according to an individual's age, size and degree of pneumatization of the maxillary sinus and the state of dental retention [4].

The apices of the maxillary posterior teeth roots may protrude into the sinus, therefore the thickness of sinus floor is markedly reduced. After extraction, some complications might occur, like oroantral fistulae or root displacement, especially for first and second maxillary molars. Among them, the first molar, being the first permanent tooth to erupt, is frequently prematurely extracted, exposing the maxillary sinus to the risk of oroantral communication.

The primary aim of the study was to assess the distances between the apices of the first molar roots and the floor of the maxillary sinus, expressed as the thickness of the bony plate between the root apices and the antrum, 
with aids of the cone beam computed tomography (CBCT) images. In addition, the distance between the furcation area and the sinus floor, its clinical relevance, as well as age-related changes of the measurements, were evaluated. The null hypothesis that the means of measurements do not differ significantly between age groups, was formulated in the present study.

\section{PATIENTS AND METHODS}

The study was conducted between January and December 2011. The dental CBCT images were taken from 97 patients. The images and the demographic data were obtained from the archives belonging to the Clinic of Oral Implantology, "Dr. Carol Davila" Central Universitary Emergency Military Hospital, Bucharest, Romania. The study was approved by the Ethical Committee for Research from the University of Medicine and Pharmacy, Bucharest.

Only maxillary first molars without apical periodontal diseases and/or without periodontal pockets were included in the study. One maxillary first molar was selected from each patient. The patients had no evidence of maxillary sinus inflammation.

All subjects were scanned using a CBCT machineiCat (Imaging Sciences International), and the CT data were analyzed using dedicated software-iCatVision. All studies were obtained using the same scanning protocol: sensor dimension-20 $2025 \mathrm{~cm}$; grayscale resolution$14 \mathrm{bit}$; voxel dimension $-0.25 \mathrm{~mm}$; acquisition time13.9 seconds; $120 \mathrm{KV}, 5 \mathrm{~mA}$; number of acquired images -528 .

All subjects were similarly positioned, with the occlusal plane in close proximity to the horizontal positioning laser beam, which was parallel to the orbital-auricular horizontal; the vertical positioning beam was placed on the patient's mid line.

The images were examined by the same oral radiology and implantology specialist (MS). Lines were drawn on the cross-sectional images between the deepest point of the maxillary sinus floor and the root tips of the maxillary first molar, and the distances were measured using built-in measurement tools. In order to accurately position the axial cross-section images at the root apex the distance between two consecutive sections was set at $0.25 \mathrm{~mm}$.

Images were grouped according to the size of measurements performed between the root tips and the maxillary sinus floor, as follows: class 0 : distance $(\mathrm{d})=0 \mathrm{~mm}$; class 1: $0 \mathrm{~mm}<\mathrm{d}<2 \mathrm{~mm}$; class $2: 2 \mathrm{~mm} \leq \mathrm{d}<4 \mathrm{~mm}$; class 3: $4 \mathrm{~mm} \leq \mathrm{d}<6 \mathrm{~mm}$; class $4: 6 \mathrm{~mm} \leq \mathrm{d}$ (Figures 1-5).

\section{Statistical Analysis}

Data distributions were expressed as means, standard

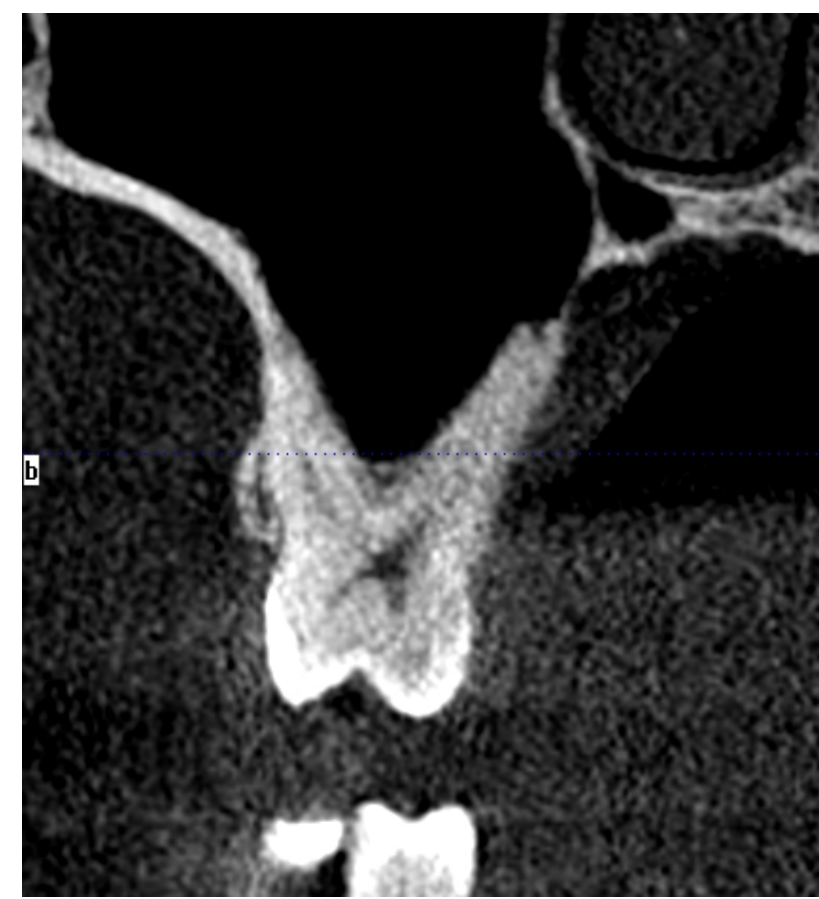

Figure 1. Class 0 relationship between the palatal root and the sinus floor.

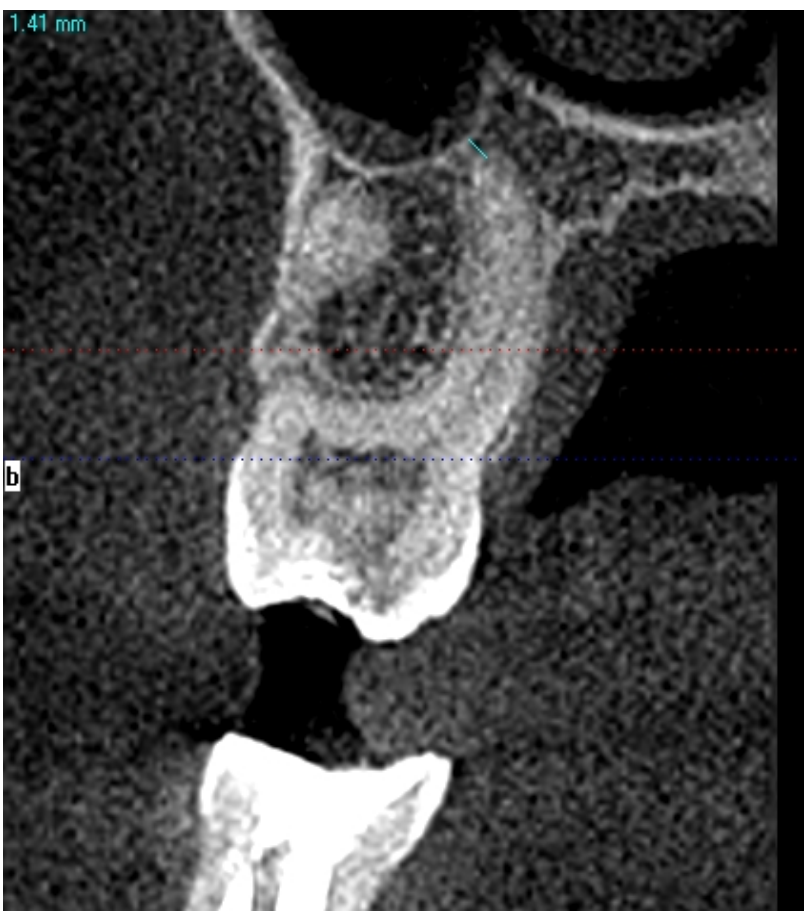

Figure 2. Class 1 relationship between the palatal root and the sinus floor (1.41 $\mathrm{mm}$ distance).

deviations (SD), ranges, and percentages, as appropriate.

The Spearman's Rank Correlation coefficient was used to test the univariate associations between furcation-sinus floor distance and each root class. Multiple linear regression models were used to examine these associations. 


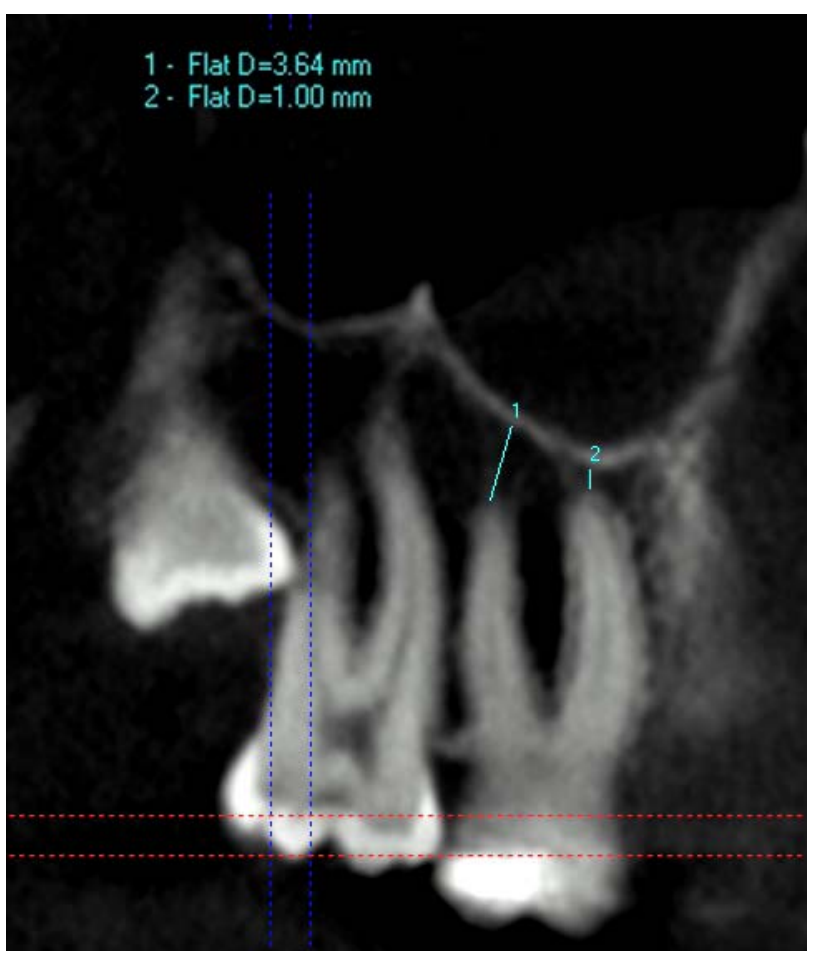

Figure 3. Class 1 relationship between the mesio-buccal root and the sinus floor $(1.00 \mathrm{~mm})$. Class 2 relationship between the disto-buccal root and the palatal floor $(3.64 \mathrm{~mm})$.

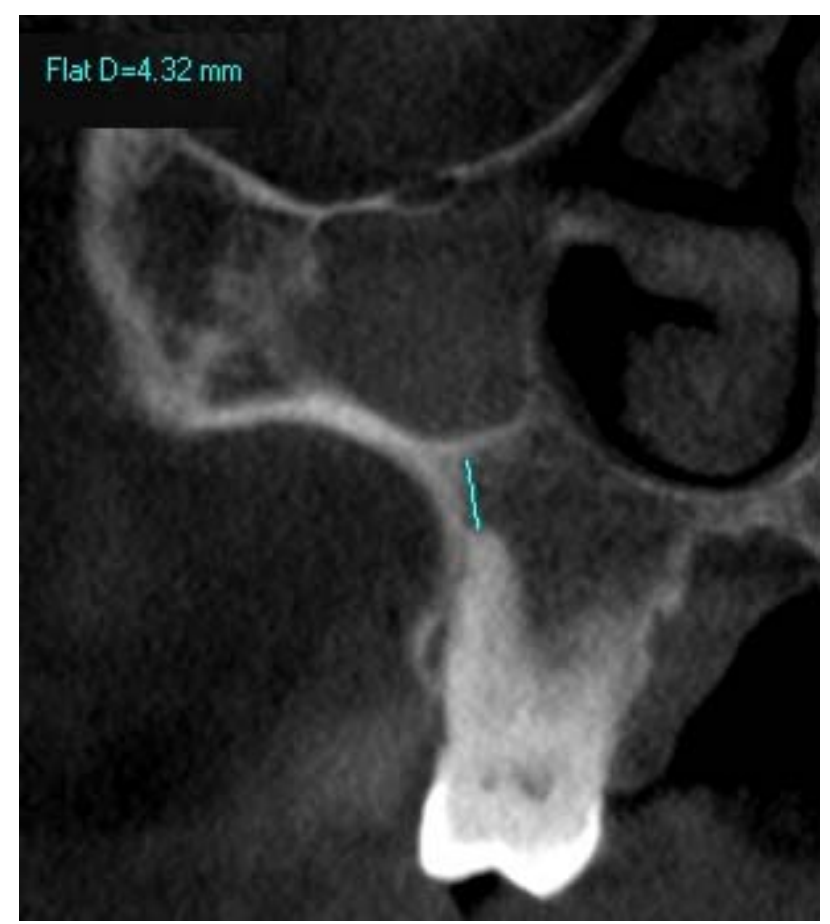

Figure 4. Class 3 relationship between the mesio-buccal root and the sinus floor $(4.32 \mathrm{~mm})$.

The Student $t$-test was used to compare the differences between the mean distances in males versus females. One-way ANOVA (Bonferroni correction) was used to

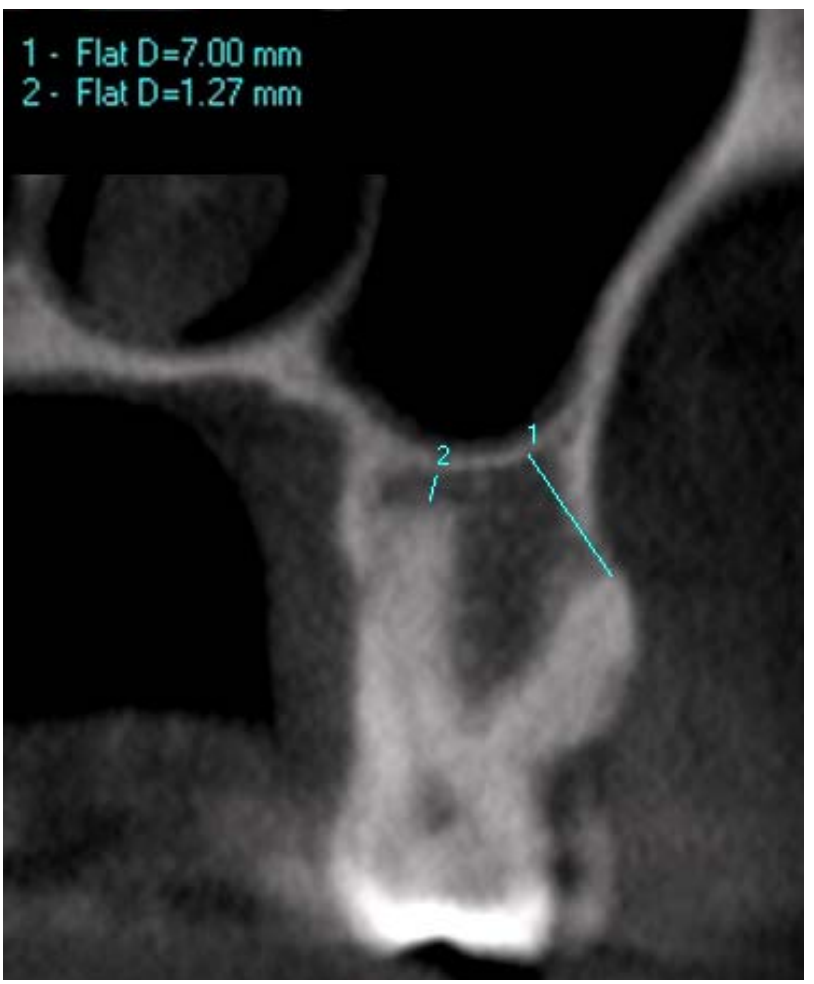

Figure 5. Class 1 relationship between the palatal root and the sinus floor $(1.27 \mathrm{~mm})$. Class 4 relationship between the distobuccal root and the sinus floor $(7.00 \mathrm{~mm})$.

test the null hypothesis. All tests of significance were 2-tailed.

The data were analysed statistically on the computer using StataIC 11 (StataCorp. 2009. Stata: Release 11. Statistical Software. College Station, TX, USA). A $p$ value $<0.05$ was considered statistically significant.

\section{RESULTS}

Fifty-two males, mean age of 38.83 years (SD 11.5, range 20 to 64), and 45 females, mean age of 38.11 (SD 11.89 , range 24 to 67 ) were recruited for this study. The main outcomes of the measurements are shown in Table 1.

The prevalence of class 0 was the highest for the palatal root, followed in descending order by mesio-buccal and disto-buccal roots (Table 2). The means of furcation-sinus floor distances depending on each root class are presented in Table 3.

The gender did not influence significantly the mean values of the measured distances ( $t$-test, $p>0.05$ ).

\subsection{Assessment of Possible Associations between Furcation-Sinus Floor Distance and Root Classes}

The highest correlation coefficient was recorded when assessing the relationship between furcation-sinus floor distance and palatal root classes $($ rho $=0.66, p<0.001$, 
Table 1. The main outcomes of distance to sinus floor measurements (mm).

\begin{tabular}{cccc}
\hline Variable & $\mathrm{N}$ & Mean (SD) & Range \\
\hline Mesio-buccal root & 97 & $1.7(2.01)$ & 0 to 9.71 \\
Disto-buccal root & 97 & $1.49(1.69)$ & 0 to 7.59 \\
Palatal root & 97 & $1.51(1.9)$ & 0 to 7.63 \\
Furcation & 97 & $6.66(3.05)$ & 2.25 to 14.9 \\
\hline
\end{tabular}

Table 2. The frequency and prevalence of first molar roots per class.

\begin{tabular}{cccc}
\hline Class & Mesio-buccal $\operatorname{root}^{*}(\%)$ & ${\text { Disto-buccal } \operatorname{root}^{*}(\%)}^{*}$ & ${\text { Palatal } \operatorname{root}^{*}(\%)}^{*}(43.33)$ \\
1 & $39(40.21)$ & $37(38.14)$ & $43(44.33)$ \\
2 & $22(22.68)$ & $29(29.9)$ & $25(25.77)$ \\
3 & $25(25.77)$ & $24(24.74)$ & $15(15.46)$ \\
4 & $7(7.22)$ & $5(5.15)$ & $11(11.34)$ \\
\hline
\end{tabular}

${ }^{*} \mathrm{~N}=97$.

Table 3. Furcation-sinus floor mean distance ( $\mathrm{mm})$ measured per root class.

\begin{tabular}{|c|c|c|c|c|c|c|c|c|c|c|}
\hline \multirow{3}{*}{ Roots } & \multicolumn{10}{|c|}{ Class } \\
\hline & \multicolumn{2}{|c|}{0} & \multicolumn{2}{|r|}{1} & \multicolumn{2}{|r|}{2} & \multicolumn{2}{|r|}{3} & \multicolumn{2}{|r|}{4} \\
\hline & $\mathrm{N}$ & Mean (SD) & $\mathrm{N}$ & Mean (SD) & $\mathrm{N}$ & Mean (SD) & $\mathrm{N}$ & Mean (SD) & $\mathrm{N}$ & Mean (SD) \\
\hline Mesio-buccal & 39 & $5.3(2.2)$ & 22 & $5.96(2.28)$ & 25 & $7.64(3.14)$ & 7 & $9.69(2.76)$ & 4 & $12.41(2.35)$ \\
\hline Disto-buccal & 37 & $4.7(1.53)$ & 29 & $6.44(2.57)$ & 24 & $8.65(2.92)$ & 5 & $10.48(3.41)$ & 2 & $12.67(0.53)$ \\
\hline Palatal & 43 & $4.88(1.68)$ & 25 & $6.19(2.26)$ & 15 & $8.4(2.6)$ & 11 & $10.95(2.85)$ & 3 & $11.78(2.28)$ \\
\hline
\end{tabular}

Table 4. The mean values of root- and furcation-sinus floor distances related to age decades.

\begin{tabular}{cccccc}
\hline \multirow{2}{*}{ Root/Furcation } & $20-29$ ys & $30-39$ ys & $40-49$ ys & $50-59$ ys & $60-69$ ys \\
\cline { 2 - 6 } & \multicolumn{5}{c}{ Mean (SD) } \\
\hline Mesio-buccal & $0.81(1.12)$ & $1.19(1.33)$ & $1.72(1.99)$ & $3.59(2.58)$ & $3.93(2.75)$ \\
Disto-buccal & $0.98(1.53)$ & $1.07(1.22)$ & $1.58(1.74)$ & $2.78(1.83)$ & $2.81(2.3)$ \\
Palatal & $0.57(1.54)$ & $1.28(1.47)$ & $1.75(2.53)$ & $2.76(1.74)$ & $3.2(1.65)$ \\
Furcation & $6.01(2.72)$ & $6.07(2.88)$ & $6.94(3.2)$ & $7.93(3.03)$ & $8.97(3.53)$ \\
\hline
\end{tabular}

$\mathrm{n}=97)$. The associations between furcation-sinus floor distance and palatal and disto-buccal root classes maintained significant after including them in a multivariate model (95\% CI of adjusted analyses: 0.87 to 1.79 and 0.46 to 1.51 , respectively; $p<0.001)$.

\subsection{Results Stratification Based on Age Decades}

In order to assess whether the bone thickness may be influenced by age variations, the measurements were stratified based on age decades. Five groups were obtained. There was a trend of increasing distance between root apices and sinus floor in older age groups $(p<$ $0.005)$. The highest variations of distances to sinus floor were recorded for the mesio-buccal root, followed by the palatal and disto-buccal roots (Table 4).

Although the distance furcation-sinus floor had the same increasing trend, no statistical significant differences were recorded between age groups.

\section{DISCUSSION}

The study aimed to assess the distance between the apices of the first molar roots and the floor of the maxillary sinus, expressed as the thickness of the bony plate between the root apices and the antrum, with aids of the CBCT images. Additionally, the age-related changes of the measurements were evaluated. With respect to the results obtained, the null hypothesis was rejected.

Among the recent radiographic techniques, the method used in the present study, that is CBCT, is valuable for implant treatment planning and placement due to the $3 \mathrm{D}$ imaging of hard tissue structures, being appropriate for evaluation of sinus floor integrity after tooth extraction and before impression techniques [5].

The highest number of cases with classes 0 and 1 was recorded for the palatal root, and the disto-buccal root, respectively. Anatomically, three teeth are described as being in close proximity of the sinus floor: the second 
maxillary premolar, the first maxillary molar, and the second maxillary molar. Among them, the first maxillary molar roots were emphasized as having a direct relationship with the maxillary sinus floor. The roots of the second molars (especially the mesio-buccal roots), followed by the first molars (especially the buccal roots), third molars, second premolars and first premolars have the closest proximity to the sinus floor [6]. Based on CT analyses, the distance of root apices to the floor of the maxillary sinus was reported to be the smallest in the mesio-buccal root of the second molar among the maxillary posterior teeth [7]. In another study, the distance between sinus floor and root tip was the longest for the first premolar root tip and the shortest for the second molar disto-buccal root tip for both right and left sides. In the same study, the maxillary first molar was reported to have the shortest distance for the disto-buccal root tip, whilst the longest was recorded for the palatal root [8]. Interestingly, in the present study, the mesio-buccal root of the maxillary first molar was over passed by the palatal root (61 cases versus 68 cases with classes 0 and I), although the high prevalence of class 0 recorded for all three roots confirms the close anatomical relationships between the tooth and the sinus floor. Georgescu et al. [9] have recently obtained higher mean values when measuring the distances between the root apices of the maxillary first molar and the sinus floor, and this can be explained by the lack of cases with class 0 . In the current study, the prevalence of class 0 cases was around $40 \%$.

The vertical relationships between the roots of maxillary teeth and the sinus inferior wall have been classified before. Thus, Freisfeld et al. [10] suggested three types of vertical relationships (class 0: teeth roots not contact the sinus floor; class 1: teeth roots contact the sinus floor, but not project on sinus cavity; class 2 : teeth roots project into the sinus cavity), while other studies [11,12] described five vertical classifications (class 0 : teeth roots do not contact the sinus floor; class 1: teeth roots contact the sinus floor, but do not project into the sinus cavity; class 2: buccal roots project into the sinus cavity; class 3 : palatal roots project into the sinus cavity; class 4 : buccal and palatal roots project into the sinus cavity).

In order to evaluate the furcation topography, we suggest here a new classification of the vertical relationships, based on the thickness of the bony plate. When assessing the mean distance from the root furcation to the sinus floor, the present findings indicate that if the maxillary first molar is in relationship with the maxillary sinus floor, belonging to classes 2,3 , or 4 , the lowest average values of the bone thickness between the furcation and the sinus floor are $7.64 \mathrm{~mm}, 9.69 \mathrm{~mm}$ and $12.41 \mathrm{~mm}$, respectively. In such cases, after tooth extraction, the remaining bone height allows the clinician to insert an implant immediately after extraction, without the need of sinus floor elevation. The mean values of the bone thickness between furcation and sinus floor recorded in classes 0 and 1 , recommend caution not only in root canal treatments (RCT), but also in extraction and immediate implant therapy.

The natural morphology of the root canal is tapered, with an increase of its diameter from the apical foramen to the coronal orifice. The most critical area is the apical zone of the root canal, mainly due to its complex morphology. It consists of lateral canals, accessory canals, apical deltas, apical ramifications, isthmi, areas of resorption etc. Particularly, the apical area of the maxillary first molar is in the close vicinity of the maxillary sinus. During RCT, the apical patency of the canal is obtained by inserting a small diameter endodontic file through the apical foramen. With regards to these procedures, there is a high risk of perforating the apical tissues as well as the sinus floor with the file, in class 0 relationships. It has been reported a case of orbit abscess after RCT of a maxillary first molar, caused by a rapid exacerbation of periapical inflammation [13].

When planning endodontic surgery of the first maxillary molar (e.g. apicoectomy), one should also take into consideration the oroantral communication that may occur. It has been proven that conventional periapical radiographs cannot be used as predictors for perforation of the maxillary sinus during periapical surgery of maxillary premolars and molars [14]. Therefore, in such cases, preoperative CBCT scan is highly recommended.

The close proximity between the first molar roots and the floor of the sinus represents an issue not only for endodontic procedures, but also for periodontal surgery and dental prosthetic therapy. Huang and Brunsvold [15] reported a case of maxillary sinusitis following the periodontal treatment of maxillary first molar, with deep pockets and major bony defects. There is a case report describing that after a maxillary molar extraction, the impression material was pushed into the antrum through an unseen oroantral fistula [5].

The mean distances between the root apices and sinus floor increased with age. Regarding the volume of the maxillary sinus, it has been demonstrated that this increases up to the age of 20 years, but then it starts decreasing [16]. Another study revealed that the growth of the maxillary sinus continues until the 3 rd decade in males [17]. In this framework, our results suggest that the teeth not only represent a good support for maintaining the sinus bone floor, but also, if healthy, may stimulate bone apposition throughout life. The results of the furcation-sinus floor distances, although higher in the last decade groups, did not show the same variation. Follow-up studies would provide more details about the age related distance variations and the reasons why some parameters are more affected than others. 


\section{CONCLUSION}

Altogether, the above results suggest that all roots of the maxillary first molar are in close proximity of the sinus floor. Among them, not only had the palatal root the closest relationship with the sinus floor, but also proved to be the best predictor for the furcation-sinus floor distance. The bone thickness measured between the furcation area and the sinus floor may be a valuable indicator in implantology. The clinician should be aware of the anatomical and morphological details of this root, especially when taking surgical decisions.

\section{ACKNOWLEDGEMENTS}

The study was supported by the Sectoral Operational Program Human Resources Development (SOP HRD), financed by the European Social Fund and by the Romanian Government under the contract number POSDRU/89/1.5/S/64109 (author \#3).

\section{REFERENCES}

[1] Waite, D.E. (1971) Maxillary sinus. Dental Clinics of North America, 15, 349-368.

[2] Lambrecht, J., Böhlck, I. and Dierck, P. (1986) Ist die Kieferhöhle physiologischerweise keimfrei? In: Watzek, G. and Matejka, M., Eds., Erkrankungen der Kieferhöhle, Springer, Berlin, 31-37. doi:10.1007/978-3-7091-8833-0_4

[3] Watzek, G., Bernhart, T. and Ulm, C. (1997) Complications of sinus perforations and their management in endodontics. Dental Clinics of North America, 41, 563-583.

[4] Laine, F. (1999) Diagnostic imaging of the maxillary sinus. Oral and Maxillofacial Surgery Clinics of North America, 11, 45-67.

[5] Dimitrakopoulosand, I. and Papadaki, M. (2008) Foreign body in the maxillary sinus: Report of an unusual case. Quintessence International, 39, 698-701.

[6] Abubaker, A.O. (1999) Applied anatomy of the maxillary sinus. Oral and Maxillofacial Surgery Clinics of North America, 11, 1-13.

[7] Eberhardt, J.A., Torabinejad, M. and Christiansen, E.L. (1992) A computed tomographic study of the distances between the maxillary sinus floor and the apices of the maxillary posterior teeth. Oral Surgery, Oral Medicine, Oral Pathology, 73, 345-346. doi:10.1016/0030-4220(92)90133-B

[8] Kilic, C., Kamburoglu, K., Yuksel, S.P. and Ozen, T. (2010) An assessment of the relationship between the maxillary sinus floor and the maxillary posterior teeth root tips using dental cone-beam computerized tomography. European Journal of Dentistry, 4, 462-467.

[9] Georgescu, C.E., Rusu, M.C., Sandulescu, M., Enache, A.M. and Didilescu, A.C. (2012) Quantitative and qualitative bone analysis in the maxillary lateral region. Surgical and Radiologic Anatomy, 34, 551-558. doi:10.1007/s00276-012-0955-6

[10] Freisfeld, M., Drescher, D., Schellmann, B. and Schüller, H. (1993) The maxillary sixth-year molar and its relation to the maxillary sinus. A comparative study between the panoramic tomogram and the computed tomogram. Fortschritte der Kieferorthopädie, 54, 179-186. doi:10.1007/BF02341464

[11] Kwak, H.H., Park, H.D., Yoon, H.R., Kang, M.K., Koh, K.S. and Kim, H.J. (2004) Topographic anatomy of the inferior wall of the maxillary sinus in Koreans. International Journal of Oral and Maxillofacial Surgery, 33, 382-388. doi:10.1016/j.ijom.2003.10.012

[12] Sharanand, A. and Madjar, D. (2006) Correlation between maxillary sinus floor topography and related root position of posterior teeth using panoramic and crosssectional computed tomography imaging. Oral Surgery, Oral Medicine, Oral Pathology, Oral Radiology, and Endodontics, 102, 375-381. doi:10.1016/j.tripleo.2005.09.031

[13] Koch, F., Breil, P., Marroquín, B.B., Gawehn, J. and Kunkel, M. (2006) Abscess of the orbit arising $48 \mathrm{~h}$ after root canal treatment of a maxillary first molar. International Endodontic Journal, 39, 657-664. doi:10.1111/j.1365-2591.2006.01130.x

[14] Oberli, K., Bornstein, M.M. and von Arx, T. (2007) Periapical surgery and the maxillary sinus: Radiographic parameters for clinical outcome. Oral Surgery, Oral Medicine, Oral Pathology, Oral Radiology, and Endodontics, 103, 848-853. doi:10.1016/j.tripleo.2006.09.017

[15] Huangand, C.H. and Brunsvold, M.A. (2006) Maxillary sinusitis and periapical abscess following periodontal therapy: A case report using three-dimensional evaluation. Journal of Periodontology, 77, 129-134. doi:10.1902/jop.2006.77.1.129

[16] Ariji, Y., Kuroki, T., Moriguchi, S., Ariji, E. and Kanda, S. (1994) Age changes in the volume of the human maxillary sinus: A study using computed tomography. Dento Maxillo Facial Radiology, 23, 163-168.

[17] Jun, B.C., Song, S.W., Park, C.S., Lee, D.H., Cho, K.J. and Cho, J.H. (2005) The analysis of maxillary sinus aeration according to aging process; volume assessment by 3-dimensional reconstruction by high-resolutional CT scanning. Otolaryngology and Head and Neck Surgery, 132, 429-434. doi:10.1016/j.otohns.2004.11.012 